\title{
Research of Social Network Information Propagation Model Based on Public Interest and 0pinion
}

\author{
Juntao Li, Tingting Dong, Meng Li \\ Graduate School, Beijing Wuzi University, Beijing, China \\ Email: $1174486142 @ q q . c o m$
}

Received 22 March 2016; accepted 25 April 2016; published 28 April 2016

Copyright (C) 2016 by authors and Scientific Research Publishing Inc.

This work is licensed under the Creative Commons Attribution International License (CC BY).

http://creativecommons.org/licenses/by/4.0/

\section{(c) (i) Open Access}

\begin{abstract}
During the diffusion of information on the network, the users generally have such an experience that at the beginning they get aware of the news they never know and may be willing to inform others, then their interests fade away, finally the information stops flowing. Meantime, their points of view are changing as the networks' structure changes. Therefore, this article brings in the interest attenuation mechanism and the social networks consensus evolution mechanism on the basic of the improved SEIR model. So to begin with, we establish a model to analyze how users' interests change during the diffusion of information on the network. Second we establish another model to analyze the evolution of the opinion during the diffusion of information on the network. At last, we establish a final model by using dynamic equations adding the results of the two models above.
\end{abstract}

\section{Keywords}

Propagation, Social Network, SEIR

\section{Introduction}

Information sharing is the basis of human society. A History of Communications advances a new theory of media that explains the origins and impact of different forms of communication on human history [1]. In the process of information propagation, the interests attenuation produces much effects on the transmission of information online. Nowadays, it has become a common phenomenon that a lot of things are done through the internet. Obviously incomputable information will come into being and affects people's daily life. Human beings, as a special existence on earth, connect with each other in different ways with their independence kept at the same time. Hence, people may have different opinions toward same problem, and their own opinions do the 
decision work whether the message is worth spreading or not. When one person faces a message that has already been republished several times, two factors (one is his own interest and another is the amount of this message's followers) work together. At the time that he loses interest in the message, he will stop diffusing it. Through this process the inclination toward repulsion stands out. In order to quantify the impact that the first line says in this section, we turn to mathematic methods. We analyzed the problem and then established several equations to denote it logically and reasonably.

In our paper we establish two submodels and one master model based on SEIR model to solve this problem. First we establish an interest attenuation model and the main innovative point is that we use the coefficient of interest attenuation $\zeta$ to quantify interest attenuation. Then we establish an opinion evolution model and the main innovative point is that we use the reinforcement effect of opinions $\zeta$ to quantify opinion intensity. At last we use Matlab to simulate our model. By analyzing the how two factors influence information propagation respectively and comparing the influence of the two factors on information propagation, we get some creative conclusions.

\section{Basic Assumptions}

- Assumption 1. The users' interests do not couple with their opinions.

- Assumption 2. We regard the information attenuation characteristics as users' interests attenuation characteristics. So the probability of interests attenuation can be denoted by $\mathrm{P}$ (the transmission probability of interest):

$$
\zeta=1-p
$$

where $p$ is the transmission probability of interest. It is also the function of information's value, mode of propagation and the influence of node.

- Assumption 3. There is only one source node in the network with others all healthy nodes at the initial state. At the beginning, every node has its own original opinion, and the distribution of these opinions is uniform.

- Assumption 4. We assume that D represents the inclination of every node's opinion, and D has three options including support, neutrality and objection. $T$ is used to stand for the intensity of the inclination. $T$ ranges from zero to one. Much closer to one, the $T$ is means the degree of support intensity gets higher [1].

\section{Model Construction and Analysis}

\subsection{Interest Attenuation Model}

\section{Step 1. Characteristic extraction [2]}

Influence of node: the sum of all the messages' influence that are released in a certain period from one node. Provided that \#RT represents the times that the messages were republished, then the influence of node can be expressed as:

$$
\operatorname{Influ}(u)=\lg (\# R T+1)
$$

Characteristic of information: We use emotional attribute Sentic $(C)$ to represent it. The message that has great emotional intensity tends to get spread more widely. Two-dimensional vector, positive and negative dimensions, can represent it. We will do some work with the eigenvectors to make the sum of all the components in the vector is one. We assume that $x$ stands for the positive emotion score, $y$ stands for the neutral emotion score and $z$ stands for the negative emotion score. Hence, the eigenvector can be expressed as:

$$
\operatorname{Sentic}(c)=\left(\frac{x}{x+z}, \frac{z}{x+z}\right)^{\mathrm{T}}
$$

The form of information communication: the form of information communication relates to the structure of the network. This article employs two nodes' p2p set Jaccard distance to denote the similarity of network structure.

$$
\operatorname{Sims}(u, v)=\frac{|N(u) \cap N(v)|}{|N(u) \cup N(v)|}
$$


$N(u)$ is the set of the neighbor node of $u, N(v)$ is the set of the neighbor node of $v$.

\section{Step 2. Model construction}

Characteristic parameter is extracted, which can better reflect Interest attenuation, combining it into the feature vector $\phi_{n}$ :

$$
\phi_{n}=\left(\begin{array}{c}
\operatorname{Influ}(u) \\
\operatorname{Sentic}(c) \\
\operatorname{Sim}-s(u, v)
\end{array}\right)
$$

The basic function can be expressed by linear combination of eigenvectors:

$$
f_{a}(u, v, c)=\alpha_{0}+\alpha_{1}^{T} \phi_{n}
$$

$\alpha_{0}$ is a constant, $\alpha_{1}^{T}$ is the weight of every characteristic.

Transmission probability can be calculated by Bayesian logistic function [3]:

$$
p(u, v, c)=\frac{1}{1+\exp \left\{-f_{\alpha}(u, v, c)\right\}}
$$

Therefore, the probability of interest attenuation is:

$$
\zeta=1-p(u, v, c)=1-\frac{1}{1+\exp \left\{-f_{\alpha}(u, v, c)\right\}}
$$

\subsection{Opinion Evolution Model}

\section{Step 1. Propagation model based on the modified SEIR}

This model is similar to model 1, but the exposed will be influenced by interest attenuation and other users' opinions. In addition, information spreads on the non-uniform complex network. So we can call the model ICSR so as to distinguish it from SEIR.

- the susceptible (I), who are susceptible to infection.

- the exposed (C), who are affected but in the incubation.

- the infectious (S), who are infected and have the symptom.

- the recovered (R), who recover or survive.

When users' interests in information decline, they will stop diffusing information at the rate of $\zeta$ and then enter latent state. $\zeta$ is the target value of Interest Attenuation Model. When users' opinions inclination become diversified, considering that events may have new progress, users will turn from latent state to opening state at the rate of $\zeta$ which is the target value of pinion evolution model.

\section{Step 2. Opinion transmission process based on the modified SEIR model}

According to Model 1, we employ the change of opinion intensity:

If the neighbor node $j$ belongs to $I, j$ will turn to $\mathrm{S}$ at the rate of $\beta$, and gets its opinion intensity recalculated.

$$
\begin{gathered}
S_{i}+I_{j} \stackrel{\beta}{\longrightarrow} S_{i}+S_{j} \\
T(j, t+1)=T(i, t)
\end{gathered}
$$

At the same time, node $j$ probably refuse to forward the message to others, and turn into immune state at the rate of $\alpha$. Then, there will not be any difference in two nodes' opinion intensity:

$$
S_{i}+I_{j} \stackrel{a}{\longrightarrow} S_{i}+R_{j}
$$

If the neighbor node $j$ belongs to $S$, node $i$ will stop diffusing message because of the decline of interest and turn into state $\mathrm{C}$ at the rate of $\zeta$, while node $j$ stay the same. The opinion intensity of node $j$ will get updated:

$$
S_{i}+S_{j} \stackrel{\zeta}{\longrightarrow} C_{i}+S_{j}
$$




$$
T(j, t+1)=\frac{T(i, t)+T(j, t)}{2}
$$

If the neighbor node $j$ stays at state $C$, considering that two nodes have different opinions on the same topic and will both trace the development of the event as the event may produce some new progresses, therefore, by the next point of time node $j$ will become a transmitter at the rate of $\zeta$, while both node $i$ and node $j$ will get their opinion intensity recalculated:

$$
\begin{gathered}
S_{i}+C_{j} \stackrel{\xi}{\longrightarrow} S_{i}+S_{j} \\
T(i, t+1)=\frac{T(i, t)+T(j, t)}{2} \\
T(j, t+1)=\frac{T(i, t)+T(j, t)}{2}
\end{gathered}
$$

If the neighbor node $j$ belongs to $R$, node $i$ will turn into state $R$ at the rate of $\gamma$ and both nodes' opinion intensity stay the same:

$$
S_{i}+R_{j} \stackrel{\gamma}{\longrightarrow} R_{i}+R_{j}
$$

\section{Step 3. The quantification of the effect of opinion intensification}

According to Deffuant opinion propagation model, index $T$ is used as opinion value. The difference in the inclination of opinions between different nodes decides that the nodes will turn into transmission state again or not. So, in this article $\Delta T=\left|T_{i}-T_{j}\right|$ is utilized as an intensity factor of opinion. According to the process of opinion transmission, the characteristics of opinion intensification will finally show up on the probability of network transmission. We establish an equation as below to denote the effect of opinion intensification.

$$
\xi=\frac{\beta}{1-\Delta T}
$$

\subsection{Information Propagation Model Based on Users' Interests and Opinion Evolution}

Based on the results of two models above, it has been taken into consideration that the change of users' interests and opinions may affect the process of information propagation. Then we add these two factors into the dynamic equations of information propagation. As the information propagation network can be regarded as non-uniform complex network, these kinetics equations are established on the basic that epidemic is in the non-uniform complex network.

The dynamic equations have been given [4] (Online social network propagation modeling)

$$
\begin{gathered}
\frac{\partial \rho^{i}(k, t)}{\partial t}=-k(\alpha+\beta) \rho^{i}(k, t) \sum_{k^{\prime}} P^{i}\left(k^{\prime} / k\right) \rho^{s}\left(k^{\prime}, t\right) \\
\frac{\partial \rho^{s}(k, t)}{\partial t}=k \rho^{i}(k, t) \beta \sum_{k^{\prime}} P^{i}\left(k^{\prime} / k\right) \rho^{s}\left(k^{\prime}, t\right)-k \rho^{s}(k, t) \gamma \sum_{k^{\prime}} P^{i}\left(k^{\prime} / k\right)\left(\rho^{s}\left(k^{\prime}, t\right)+\rho^{c}\left(k^{\prime}, t\right)+\rho^{r}\left(k^{\prime}, t\right)\right) \\
-\rho^{s}(k, t) \zeta+k \rho^{c}(k, t) \xi \sum_{k^{\prime}} P\left(k^{\prime} / k\right) \rho^{s}\left(k^{\prime}, t\right) \\
\frac{\partial \rho^{c}(k, t)}{\partial t}=-k \rho^{c}(k, t) \xi \sum_{k^{\prime}} P\left(k^{\prime} / k\right) \rho^{s}\left(k^{\prime}, t\right)+\rho^{s}(k, t) \zeta \\
\frac{\partial \rho^{r}(k, t)}{\partial t}=k \rho^{i}(k, t) \alpha \sum_{k^{\prime}} P^{i}\left(k^{\prime} / k\right) \rho^{s}\left(k^{\prime}, t\right)-k \rho^{s}(k, t) \gamma \sum_{k^{\prime}} P\left(k^{\prime} / k\right)\left(\rho^{s}\left(k^{\prime}, t\right)+\rho^{c}\left(k^{\prime}, t\right)+\rho^{r}\left(k^{\prime}, t\right)\right)
\end{gathered}
$$

In addition, in the interest attenuation model and opinion evolution model, we can obtain that 


$$
\begin{aligned}
\zeta=1-p(u, v, c) & =1-\frac{1}{1+\exp \left\{-f_{\alpha}(u, v, c)\right\}} \\
\xi & =\frac{\beta}{1-\Delta T}
\end{aligned}
$$

So we can obtain the characteristic of information propagation on the basic of users' interests and opinions by building simultaneous equations with this equation and four equations proposed above.

\section{Model Analysis}

We can learn that information value and form of the message have influence on $\zeta$. People's initial opinion, bias and the topology or strength of the information network have influence on $\zeta$. In addition the size of the network has influence on $\sum P\left(k^{\prime} / k\right)$. Therefore we can do simulation experiment based on this model to determine how information vdlue, people's initial opinion and bias, form of the message or its source, and the topology or strength of the information network in a region, country, or worldwide could be used to spread information and influence public opinion.

In order to explore how people's initial opinion and interests affect the topology or strength of the information network, we simulate the model by using Matalab. Then we use Originpro to process the data. The total number of nodes $\mathrm{N}$ is 10000 . The values of parameters are as follows:

$$
\alpha=0.2, \beta=0.8, \gamma=0.2
$$

\subsection{The Influence of Interest Attenuation on Information Propagation}

Here we assume $\zeta=0.2$, then we observe how information spreads with the changes in $\zeta$ which can reflects the change of public interests. And the results are as follows (see Figure 1 and Figure 2).

According to Figure 1, we can learn that as the coefficient of interest attenuation $\zeta$ increases, the number of nodes in the state of $\mathrm{R}$ which can reflects the scale of information propagation decreases. So public interests have big influence on information propagation and interest attenuation can inhibit the spread of information effectively. That is to say, the attenuation mechanism of interest has negative correlation with the scale of information propagation. This result is in accordance with the conclusion which Nekovee put forwards [5]. Figure 2 shows the process of information propagation and it also confirms that the bigger the coefficient of interest attenuation $\zeta$ is, the smaller the number of people who are more likely to spread information is.

In another hand, at the beginning or end of information propagation, information propagation is less affected by interest attenuation. But it influences information propagation probability apparently in the intermediate stages.

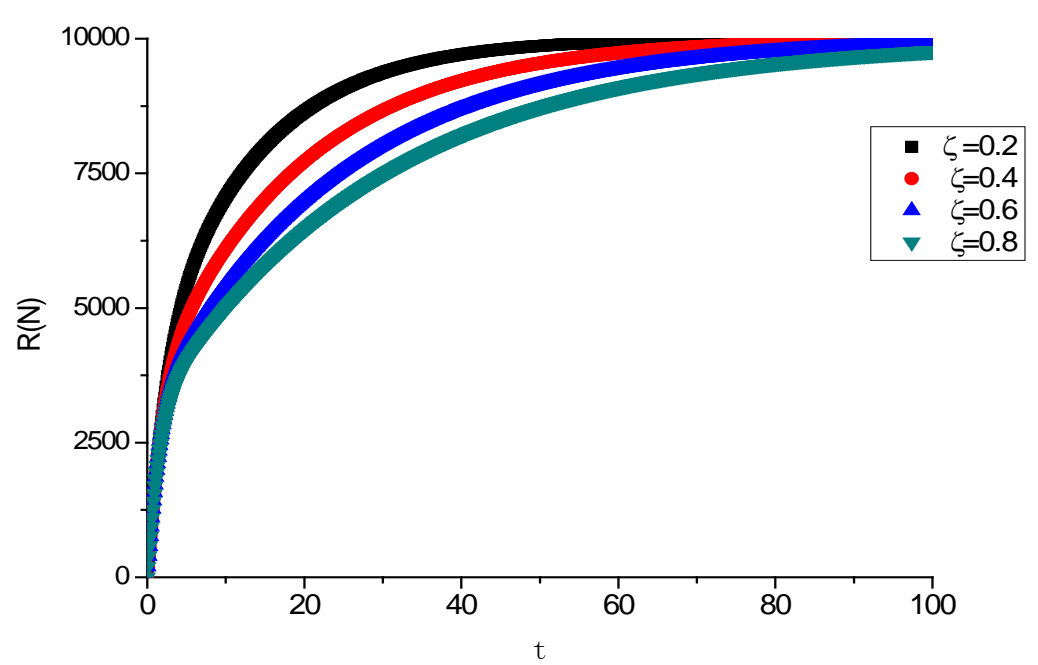

Figure 1. The number of nodes in the state of R. 


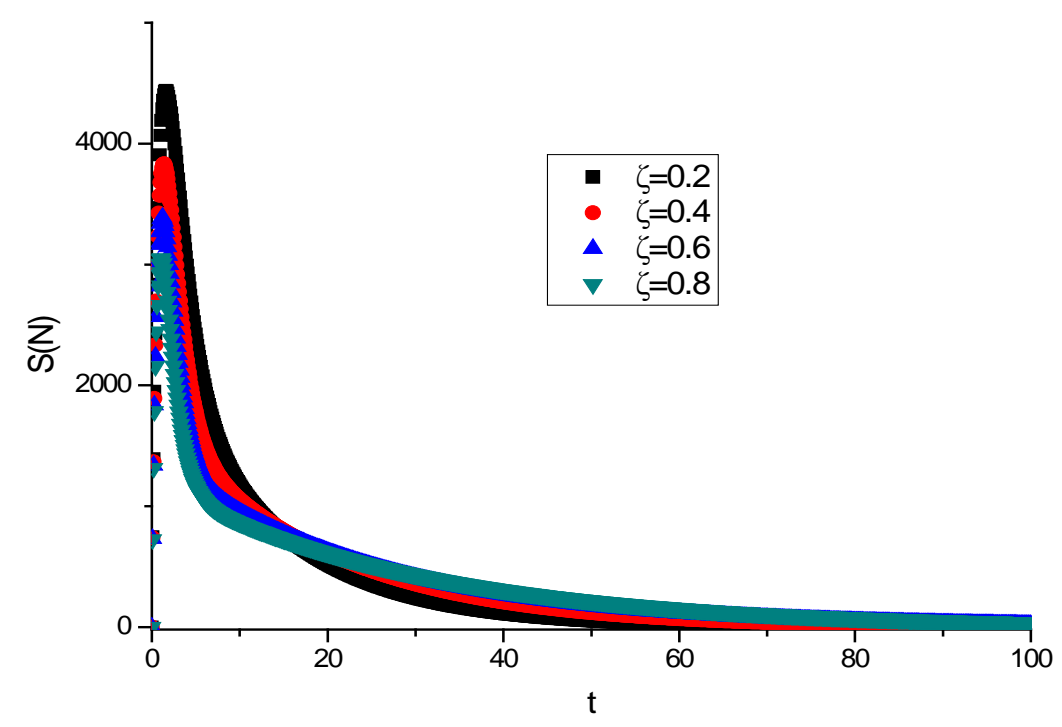

Figure 2. The number of nodes in the state of S.

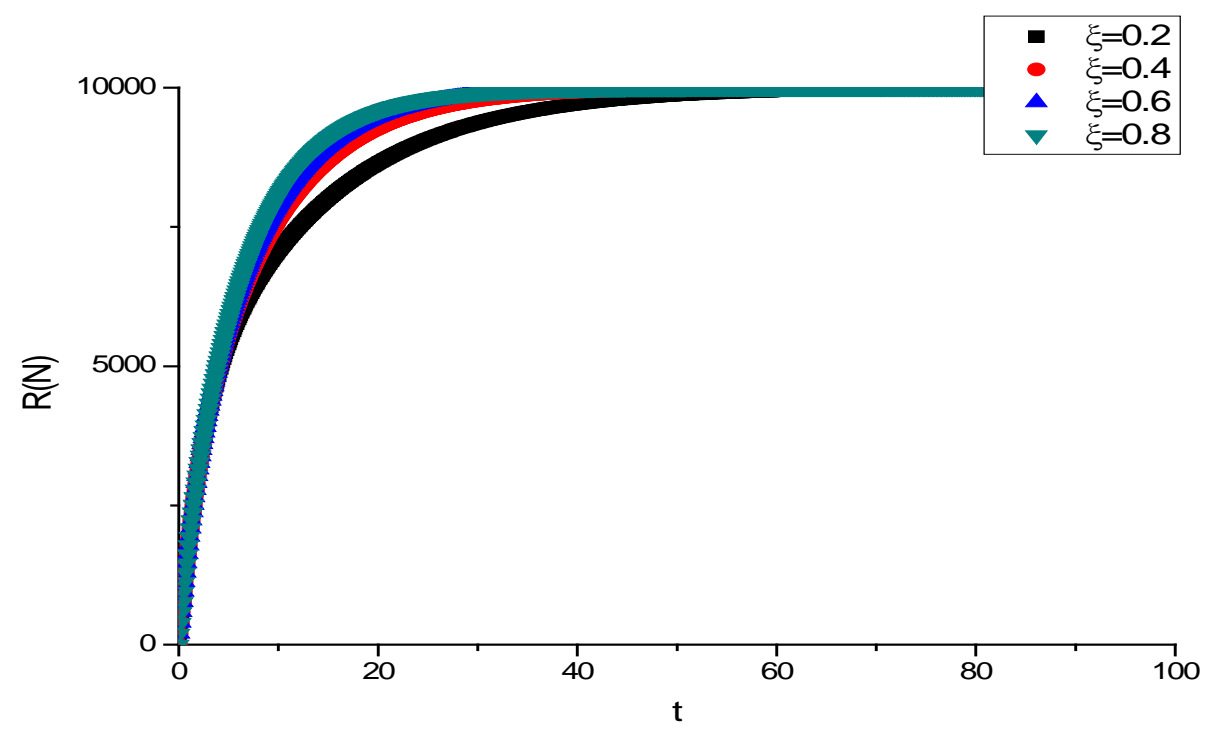

Figure 3. The number of nodes in the state of R.

\subsection{The Influence of Opinion Intensity on Information Propagation}

Here we assume $\zeta=0.2$, then we observe how information spreads with the changes in $\zeta$ which can reflects the change of public opinion intensity. And the results are as follows (see Figure 3 and Figure 4).

According to Figure 3, we can learn that as the reinforcement effect of opinions $\zeta$ increases, the number of nodes in the state of $\mathrm{R}$ which can reflects the scale of information propagation also increases. So opinion intensity has big influence on information propagation and reinforcement effect of opinions can promote the spread of information effectively. Figure 4 shows the process of information propagation and it also confirms this law. In another hand, at the beginning or end of information propagation, information propagation is less affected by opinion intensity. But it influences information propagation probability apparently in the intermediate stages.

\subsection{Comparing the Two Cases}

According to the pictures and analysis above, we can obtain that the influencing mechanisms of the two cases are opposite. At the same time, the influence of the interest attenuation is stronger than that of opinion intensity. 


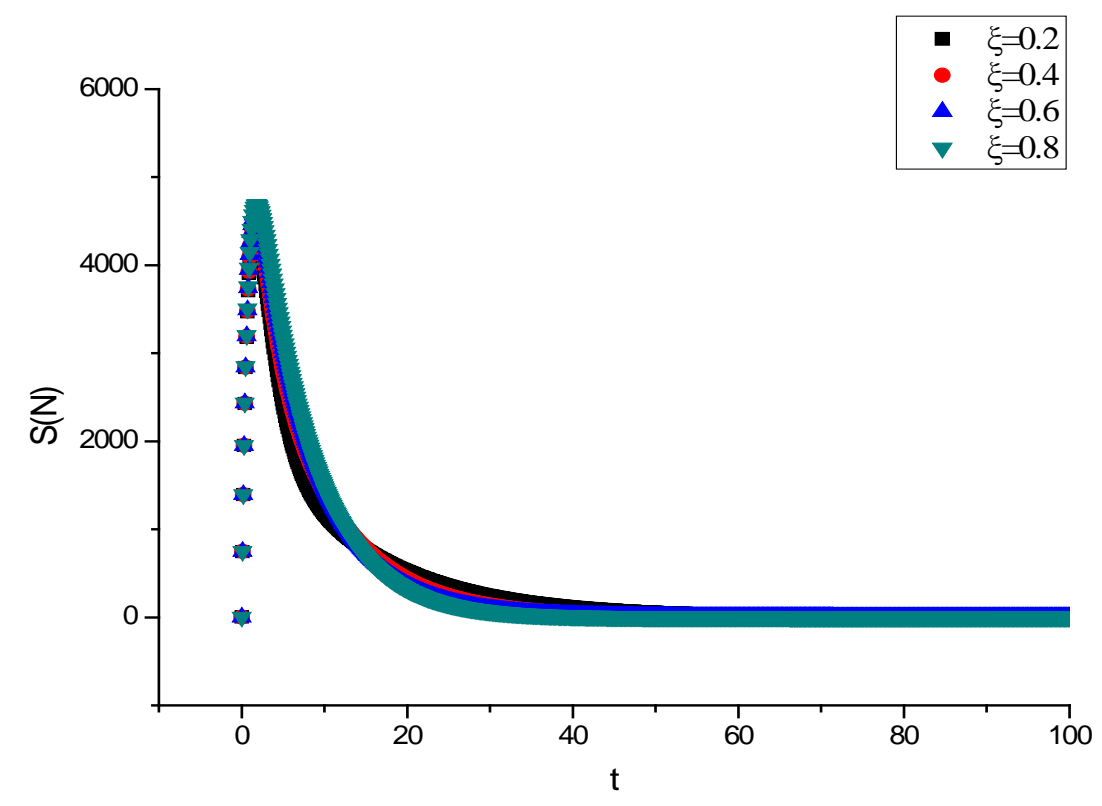

Figure 4. The number of nodes in the state of S.

\section{Conclusions}

First, the mechanisms of interest attenuation and the reinforcement effect of opinions both have great influence on information propagation and the two mechanisms are opposite.

Second, in the process of information propagation, they play different roles have different importance in different stages. So we can control information propagation by controlling the two factors in different stages.

Third, the influence of the interest attenuation is stronger than that of opinion intensity so we can control information propagation by adjusting the weights of the two factors.

\section{Acknowledgements}

This paper is supported by the Funding Project for Technology Key Project of Municipal Education Commission of Beijing (ID: TSJHG201310037036); Funding Project for Beijing key laboratory of intelligent logistics system; Funding Project of Construction of Innovative Teams and Teacher Career Development for Universities and Colleges Under Beijing Municipality (ID: IDHT20130517), and Beijing Municipal Science and Technology Project (ID: Z131100005413004); Funding Project for Beijing philosophy and social science research base specially commissioned project planning (ID: 13JDJGD013).

\section{Funding}

Funding Project for Beijing Intelligent Logistics System Collaborative Innovation Center.

\section{References}

[1] Huang, Q.H. (2014) Research on Opinion Spreading and Consensus of Complex Networks. Nanjing University of Posts and Telecommunications, Nanjing.

[2] Zhou, D.H., Han, W.B. and Wang, Y.J. (2015) A Fine-Grained Information Diffusion Model Based on Node Attributes and Content Features. Journal of Computer Research and Development, 52, 156-166.

[3] Poe, M.T. (2011) A History of Communications: Media and Society from the Evolution of Speech to the Internet. Cambridge University Press, New York.

[4] Tang, C.S. (2014) Modeling of Information Propagation in Online Social Networks and Repositing Prediction. Yanshan University, Qinhuangdao.

[5] Nekovee, M., Moreno, Y., Bianconi, G. and Marsili, M. (2007) Theory of Rumour Spreading in Complex Social Networks. Physica A: Statistical Mechanics and its Application, 374, 457-470. http://dx.doi.org/10.1016/j.physa.2006.07.017 